\title{
Effect of food intake on 92 oncological biomarkers by the Proseek Oncology II panel
}

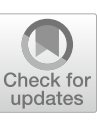

Magnus Dencker ${ }^{1 *}$ (O) Ola Björgell ${ }^{1}$ and Joanna Hlebowicz ${ }^{2}$

\begin{abstract}
Objective: To evaluates the effect of food intake on 92 oncological biomarkers to evaluate whether the timing of blood sampling could be relevant. Twenty-two healthy subjects were investigated. A total of 92 biomarkers were measured before a standardised meal as well as 30 and 120 min afterwards with the Proseek Multiplex Oncology II kit.

Results: The levels of 6 biomarkers decreased significantly $(P<0.001) 30$ min after food intake, and 4 biomarkers remained decreased $(P<0.001) 120$ min after food intake. One biomarker was significantly increased $(P<0.001)$ at both 30 and 120 min after food intake. Some changes were less than 10\%. Those biomarkers that showed a difference of more than 10\% include: Granzyme H (13\%), Methionine aminopeptidase 2 (14\%), Secretory carrier-associated membrane protein 3 (39\%), FAS-associated death domain protein (41\%), and Pancreatic prohormone (79\%). This study shows that food intake has a very modest effect on 92 different oncological biomarkers.
\end{abstract}

Trial registration National Library of Medicine trial registration number NCT01027507 (retrospectively registered on December 8, 2009)

Keywords: Proseek Oncology II, Olink

\section{Introduction}

Cancer is major cause of death and disability in the world and its prevalence is increasing [1]. The ongoing identification of novel biomarkers is critical to facilitating early detection of oncological disease. The timing of blood sampling, however, could be relevant as food digestion is known to have hemodynamic and metabolic effects [2-7]. In this study, we investigate whether certain biomarkers are affected by food intake. We have previously reported the influence of food intake on biomarkers analysed with the Proseek Multiplex CVD III, Proseek Multiplex CVD II and Proseek Multiplex Neurology I kits, and now advance our efforts with the Proseek Oncology II panel [8-10]. In this study, we assess the effect of food intake on 92 emerging oncological biomarkers. To our knowledge, this is the first study to do this.

*Correspondence: magnus.dencker@skane.se

1 Department of Medical Imaging and Physiology, Skåne University Hospital, Lund University, 20502 Malmö, Sweden

Full list of author information is available at the end of the article

\section{Main text \\ Materials and methods Study population}

The trial was registered retrospectively at the National Library of Medicine (trial registration number NCT01027507, retrospectively registered on December 8,2009 ). The study was approved by the Regional Ethical Review Board in Lund, Sweden. All subjects gave their written informed consent. The study investigated 22 healthy Caucasians (11 male and 11 female aged $25.9 \pm 4.2$ years). This is an exploratory study, first of its kind, and the data for a power calculation were not available. The data from the present study could be used for power calculations in future studies. None of the subjects took cardiovascular medication, showed symptoms of cardiovascular disease, or had a history of cardiovascular or other chronic disease. The subjects were examined between 7.30 and 11.00 after a minimum 8 -hour fast. Their height and weight was measured and body mass index (BMI) calculated. The subjects then consumed a standardised meal consisting of $300 \mathrm{~g}$ rice pudding (AXA Goda Gröten Risgrynsgröt; Lantmännen AXA, Järna, Sweden). The total caloric value of the meal was $330 \mathrm{kcal}$ : 
$10 \%$ from protein $(9 \mathrm{~g}), 58 \%$ from carbohydrates (48 g), and $32 \%$ from fat $(12 \mathrm{~g})$.

\section{Blood samples}

We collected plasma samples in EDTA test tubes before the meal, $30 \mathrm{~min}$ after the meal, and $120 \mathrm{~min}$ after the meal. We then froze the samples. No subject consumed any beverage during the experiment. We excluded one blood sample from the analysis, taken $30 \mathrm{~min}$ after the meal, as it was defective. The 92 biomarkers were then evaluated at the Olink laboratory, blinded to all other data, in Uppsala using the Proximity Extension Assay technique with the Proseek Multiplex Oncology II $96 \times 96$ reagents kit (Olink Bioscience, Uppsala, Sweden), as described previously $[11,12]$. Data are displayed as arbitrary units (AU). Values can be recalculated to actual concentrations using transformation algorithms on the Olink Bioscience website (http://www. olink.com). The conversion is just an estimate, however, and does not provide exact values. The 92 biomarkers analysed included (intra-assay variation and inter-assay variation, as supplied by the manufacturer): Alphataxilin $(7 \%, 14 \%)$, Vascular endothelial growth factor A (8\%,13\%), Carboxypeptidase E $(9 \%, 16 \%)$, Kallikrein-13 (7\%,16\%), Carcinoembryonic antigen-related cell adhesion molecule $1(5 \%, 13 \%)$, Mesothelin $(7 \%, 21 \%)$, Tumor necrosis factor ligand superfamily member $13(8 \%, 13 \%)$, Pro-epidermal growth factor $(9 \%, 11 \%)$, Tumor necrosis factor receptor superfamily member $6 \mathrm{~B}(9 \%, 13 \%)$, Syndecan-1 (8\%,15\%), TGF-beta receptor type-2 $(8 \%, 15 \%)$, Interleukin-6 (8\%,12\%), CD48 antigen $(7 \%, 14 \%)$, Secretory carrier-associated membrane protein $3(8 \%, 15 \%)$, T-lymphocyte surface antigen Ly- $9(6 \%, 14 \%)$, Interferon gamma receptor $1(7 \%, 15 \%)$, Integrin alpha- $\mathrm{V}(5 \%, 13 \%)$, TNF-related apoptosis-inducing ligand $(8 \%, 12 \%)$, Kallikrein-11 (8\%,14\%), Glypican-1 (8\%,14\%), Tissue factor pathway inhibitor $2(9 \%, 13 \%)$, Kallikrein- $8(7 \%, 14 \%)$, Vascular endothelial growth factor receptor $2(6 \%, 19 \%)$, Ly6/ PLAUR domain-containing protein $3(6 \%, 13 \%)$, Podocalyxin $(5 \%, 16 \%)$, Protein S100-A4 (7\%,14\%), Insulin-like growth factor 1 receptor $(8 \%, 15 \%)$, Receptor tyrosineprotein kinase erbB-2 (7\%,16\%), Receptor tyrosine-protein kinase erbB-3 $(6 \%, 16 \%)$, Stem cell factor $(7 \%, 14 \%)$, SPARC $(5 \%, 11 \%)$, Granzyme H $(8 \%, 16 \%)$, Transforming growth factor alpha $(8 \%, 14 \%)$, Furin $(7 \%, 16 \%)$, Protein CYR61 (9\%,15\%), Kallikrein-14 (9\%,15\%), FAS-associated death domain protein $(7 \%, 13 \%)$, Methionine aminopeptidase $2(7 \%, 12 \%)$, Nectin- $4(8 \%, 15 \%)$, Tumor necrosis factor ligand superfamily member $6 \mathrm{~B}(9 \%, 13 \%)$, Ephrin type-A receptor $2(8 \%, 15 \%)$, Integrin beta-5 $(7 \%, 13 \%)$, Galectin-1 (5\%,15\%), Seizure 6-like protein $(7 \%, 15 \%)$, Transmembrane glycoprotein NMB (5\%,16\%), Carbonic anhydrase 9 (8\%,15\%), Melanoma-derived growth regulatory protein $(7 \%, 12 \%)$, Cathepsin L2 (9\%,11\%), CD27 antigen (7\%,13\%), Xaa-Pro aminopeptidase 2 $(6 \%, 15 \%)$, Receptor tyrosine-protein kinase erbB-4 $(6 \%, 15 \%)$, Hepatocyte growth factor $(8 \%, 15 \%)$, Disintegrin and metalloproteinase domain-containing protein 8 (7\%,16\%), 5'-nucleotidase (7\%,23\%), Cyclin-dependent kinase inhibitor $1(7 \%, 12 \%)$, Delta-like protein 1 $(8 \%, 15 \%)$, Midkine $(10 \%, 15 \%)$, Tyrosine-protein kinase ABL1 (8\%,18\%), Fibroblast growth factor-binding protein $1(6 \%, 13 \%)$, Toll-like receptor $3(8 \%, 16 \%)$, Tyrosine-protein kinase Lyn $(6 \%, 13 \%)$, Proto-oncogene tyrosine-protein kinase receptor Ret $(8 \%, 17 \%)$, Vimentin $(9 \%, 15 \%)$, Tumor necrosis factor receptor superfamily member 19 $(8 \%, 15 \%)$, Cornulin $(9 \%, 17 \%)$, T cell leukemia/lymphoma protein $1 \mathrm{~A}(9 \%, 15 \%), \mathrm{CD} 160$ antigen $(8 \%, 17 \%)$, Tumor necrosis factor receptor superfamily member 4 ( $8 \%, 15 \%)$, MHC class I polypeptide-related sequence A and B (6\%,17\%), WNT1-inducible-signaling pathway protein 1 (8\%,15\%), VEGF-co regulated chemokine 1 (9\%,20\%), Pancreatic prohormone $(10 \%, 16 \%)$, Protein S100-A11 (6\%,14\%), Amphiregulin (9\%,12\%), Endothelial cell-specific molecule $1(10 \%, 15 \%)$, C-type lectin domain family 4 member K $(8 \%, 14 \%)$, ICOS ligand $(6 \%, 15 \%)$, WAP fourdisulfide core domain protein 2 (7\%,13\%), C-X-C motif chemokine $13,(6 \%, 11 \%)$, Mothers against decapentaplegic homolog 5 (7\%,13\%), A disintegrin and metalloproteinase with thrombospondin motifs $15(8 \%, 15 \%)$, CD70 antigen $(7 \%, 16 \%)$, R-spondin-3 (10\%,13\%), Folate receptor gamma $(8 \%, 13 \%)$, Carcinoembryonic antigen-related cell adhesion molecule $5(5 \%, 13 \%)$, Vascular endothelial growth factor receptor $3(6 \%, 14 \%)$, Mucin-16 $(8 \%, 15 \%)$, Wnt inhibitory factor 1 (9\%,15\%), Granzyme B $(9 \%, 14 \%)$, Fc receptor-like B (8\%,14\%), Annexin A1 (8\%,16\%), Folate receptor alpha $(8 \%, 15 \%)$. One sample for R-spondin-3 and one sample for Fc receptor-like B failed to reach detection levels. In these cases the values were set at the detection level.

\section{Statistical analysis}

Data are presented as mean \pm standard deviation (SD). Statistical analyses were carried out using Statistica 12 (StatSoft Inc, Tulsa, OK, USA). Comparison between fasting values versus 30 and $120 \mathrm{~min}$ after food intake for any given biomarker was analysed for significance with the Wilcoxon matched pairs test. Statistical significance was set at $\mathrm{P}<0.001$ to counteract the problem of multiple comparisons.

\section{Results and discussion}

Six biomarkers showed a significant decrease in levels $(\mathrm{P}<0.001) 30 \mathrm{~min}$ after food intake, and of those, 4 remained significantly decreased $(\mathrm{P}<0.001) 120 \mathrm{~min}$ after food intake (Table 1). One biomarker (Pancreatic 
Table 1 Subjects' anthropometric characteristics. Values are mean \pm SD

\begin{tabular}{ll}
\hline Variable & \\
\hline Sex (male/female) & $11 / 11$ \\
Body mass (kg) & $69 \pm 10$ \\
Height $(\mathrm{cm})$ & $177 \pm 8$ \\
BMI $\left(\mathrm{kg} / \mathrm{m}^{2}\right)$ & $21.8 \pm 2.2$ \\
BSA $\left(\mathrm{m}^{2}\right)$ & $1.8 \pm 0.2$ \\
\hline
\end{tabular}

$B M I$ body mass index, $B S A$ body surface area

prohormone) showed a significant increase $(\mathrm{P}<0.001)$ both $30 \mathrm{~min}$ and $120 \mathrm{~min}$ after food intake. The changes were most often less than $10 \%$. Some biomarkers showed a difference of more than 10\%: Granzyme $\mathrm{H}$ (13\%), Methionine aminopeptidase 2 (14\%), Secretory carrier-associated membrane protein 3 (39\%), FASassociated death domain protein $(41 \%)$, and Pancreatic prohormone (79\%). A summary of our results can be found in Table 2.

To our knowledge, no study has previously evaluated the effect of food intake on plasma proteins measured by the Proseek Multiplex Oncology II kit. Our results show that of the 92 biomarkers investigated, only 9 were affected by food intake, and the changes were modest: less than $10 \%$. Standardising food intake, therefore, is generally not required when using this kit. There are several exceptions. The greatest changes in observed levels were for Granzyme H (13\%), Methionine aminopeptidase $2(14 \%)$, Secretory carrier-associated membrane protein 3 (39\%), FAS-associated death domain protein $(41 \%)$, and Pancreatic prohormone (79\%). Our results point to the need to standardise food intake when evaluating these biomarkers.

In our study, we were able to simultaneously measure 92 plasma proteins using the Proseek Multiplex Oncology II kit. The biomarkers were selected because of their oncological relevance. Twenty-one of these biomarkers are defined as cell proliferation, 11 tumorspecific, 11 general oncology, 10 angiogenesis specific, 9 immune response, 8 cell adhesion, 7 exploratory, 6 apoptosis, 5 invasion and metastasis and 4 cell differentiation. This new technology has been used in several studies [13-21], and our investigation adds methodological data to this developing field.

We observed most of the changes $30 \mathrm{~min}$ after food intake. A total of 7 biomarkers decreased, and one increased, $30 \mathrm{~min}$ after the meal. This could be due to the changes in hemodynamics. We have previously reported a $20 \%$ increase in stroke volume and a $28 \%$ increase in cardiac output $30 \mathrm{~min}$ after food intake in this cohort [4]. There was one obvious exception.
Pancreatic prohormone, also known as Pancreatic polypeptide, is secreted by the pancreatic islets of Langerhans and is known to be released by food intake, particularly fat-rich food [22]. Moreover, Pancreatic prohormone has been known to decrease both appetite and food intake [22]. Our finding is not novel concerning postprandial changes of this biomarker. We are not aware of any study that has investigated postprandial levels of Methionine aminopeptidase 2, Secretory carrier associated membrane protein 3, or FAS-associated death domain protein. Granzyme $\mathrm{H}$ is part of a group of proteases that are found mainly in cytotoxic immune cells, and has been investigated in a variety of diseases including infectious diseases, multiple sclerosis, large granular lymphocyte leukemia, lymphoma, and breast cancer [23]. Methionine aminopeptidase 2 has been suggested to have an important role in angiogenesis, which is pivotal for the progression of solid tumours [24]. Secretory carrier associated membrane protein 3 functions as a carrier to the cell surface and is also involved in other intracellular protein trafficking, and has to our knowledge only been studied on a cellular or tissue level and our findings may not be relevant [25, 26]. FAS-associated death domain protein has a known role in apoptosis, but has also been implicated in cell proliferation, cell cycle regulation, and cell development. It has also been investigated in squamous cell carcinoma of the head and neck and in non-small cell lung cancer $[27,28]$.

To our knowledge, only one study has previously assessed the impact of food intake on multiple biomarkers. Jahn et al. evaluated Kallikrein-11, Xaa-Pro aminopeptidase 2 (mAmP), Hepatocyte growth factor, Endothelial cell specific molecule 1, and mucin 16 (Cancer antigen-125) [29]. Their results, for the most part, coincided with ours: food intake showed no effect on biomarker levels. Kallikrein-11, however, was the exception. Jahn et al. found a decrease in Kallikrein-11 levels after food intake, which we did not observe. The variance could be explained by a difference in observation time and/or meal composition.

\section{Limitations}

The limitations of the study include the study population, which consisted of young, healthy Caucasian subjects. Additional studies are recommended in older healthy subjects from different ethnic groups and in patients with disease to determine whether these findings are reproducible. Moreover, the potential interaction effect of different medications should also be considered. Further research is also recommended to evaluate the effect of different diets such as high or low fat [30]. 
Table 2 Summary of findings for 92 biomarkers before, and 30 and 120 min after a standardised meal

\begin{tabular}{|c|c|c|c|}
\hline Variable & Fasting $(n=22)$ & $\begin{array}{l}30 \text { min after food } \\
\text { intake }(n=21)\end{array}$ & $\begin{array}{l}120 \text { min } \\
\text { after food intake } \\
(n=22)\end{array}$ \\
\hline Alpha-taxilin & $1.97 \pm 0.37$ & $1.84 \pm 0.42$ & $1.58 \pm 0.47$ \\
\hline Vascular endothelial growth factor A & $9.72 \pm 0.53$ & $9.64 \pm 0.56$ & $9.64 \pm 0.62$ \\
\hline Carboxypeptidase E & $2.43 \pm 0.47$ & $2.36 \pm 0.43^{*}$ & $2.24 \pm 0.62$ \\
\hline Kallikrein-13 & $3.27 \pm 0.39$ & $3.20 \pm 0.48$ & $3.22 \pm 0.42$ \\
\hline Carcinoembryonic antigen-related cell adhesion molecule 1 & $6.75 \pm 0.15$ & $6.73 \pm 0.15$ & $6.73 \pm 0.17$ \\
\hline Mesothelin & $4.61 \pm 0.53$ & $4.62 \pm 0.54$ & $4.67 \pm 0.53$ \\
\hline Tumor necrosis factor ligand superfamily member 13 & $7.19 \pm 0.28$ & $7.10 \pm 0.28$ & $7.10 \pm 0.34$ \\
\hline Pro-epidermal growth factor & $10.74 \pm 0.24$ & $10.13 \pm 0.63^{*}$ & $9.73 \pm 0.70^{*}$ \\
\hline Tumor necrosis factor receptor superfamily member $6 \mathrm{~B}$ & $3.49 \pm 0.49$ & $3.51 \pm 0.46$ & $3.42 \pm 0.72$ \\
\hline Syndecan-1 & $6.24 \pm 0.47$ & $6.17 \pm 0.50$ & $6.23 \pm 0.57$ \\
\hline TGF-beta receptor type-2 & $6.58 \pm 0.32$ & $6.47 \pm 0.30$ & $6.53 \pm 0.36$ \\
\hline Interleukin-6 & $1.03 \pm 1.16$ & $1.01 \pm 1.23$ & $1.81 \pm 1.20$ \\
\hline CD48 antigen & $6.07 \pm 0.27$ & $5.99 \pm 0.26$ & $6.03 \pm 0.40$ \\
\hline Secretory carrier-associated membrane protein 3 & $1.67 \pm 0.57$ & $1.25 \pm 0.47$ & $1.02 \pm 0.36^{*}$ \\
\hline T-lymphocyte surface antigen Ly-9 & $4.58 \pm 0.29$ & $4.52 \pm 0.28$ & $4.58 \pm 0.42$ \\
\hline Interferon gamma receptor 1 & $3.52 \pm 0.32$ & $3.48 \pm 0.33$ & $3.50 \pm 0.28$ \\
\hline Integrin alpha-V & $2.28 \pm 0.25$ & $2.27 \pm 0.26$ & $2.27 \pm 0.20$ \\
\hline TNF-related apoptosis-inducing ligand & $6.83 \pm 0.43$ & $6.82 \pm 0.40$ & $6.86 \pm 0.49$ \\
\hline Kallikrein-11 & $4.95 \pm 0.40$ & $4.97 \pm 0.38$ & $4.77 \pm 0.37$ \\
\hline Glypican-1 & $3.83 \pm 0.40$ & $3.79 \pm 0.38$ & $3.82 \pm 0.33$ \\
\hline Tissue factor pathway inhibitor 2 & $6.36 \pm 0.43$ & $6.32 \pm 0.40$ & $6.46 \pm 0.41$ \\
\hline Kallikrein-8 & $6.27 \pm 0.34$ & $6.24 \pm 0.34$ & $5.98 \pm 0.40$ \\
\hline Vascular endothelial growth factor receptor 2 & $6.38 \pm 0.18$ & $6.34 \pm 0.18$ & $6.37 \pm 0.20$ \\
\hline Ly6/PLAUR domain-containing protein 3 & $3.59 \pm 0.28$ & $3.56 \pm 0.30$ & $3.57 \pm 0.48$ \\
\hline Podocalyxin & $2.98 \pm 0.19$ & $2.99 \pm 0.18$ & $3.02 \pm 0.18$ \\
\hline Protein S100-A4 & $3.64 \pm 0.41$ & $3.63 \pm 0.52$ & $3.40 \pm 0.49$ \\
\hline Insulin-like growth factor 1 receptor & $2.92 \pm 0.29$ & $2.83 \pm 0.32$ & $2.82 \pm 0.33$ \\
\hline Receptor tyrosine-protein kinase erbB-2 & $6.31 \pm 0.24$ & $6.26 \pm 0.26$ & $6.32 \pm 0.38$ \\
\hline Receptor tyrosine-protein kinase erbB-3 & $7.19 \pm 0.24$ & $7.15 \pm 0.21$ & $7.19 \pm 0.24$ \\
\hline Stem cell factor & $8.76 \pm 0.32$ & $8.80 \pm 0.28$ & $8.79 \pm 0.29$ \\
\hline SPARC & $5.88 \pm 0.17$ & $5.85 \pm 0.18$ & $5.83 \pm 0.16$ \\
\hline Granzyme H & $2.43 \pm 0.56$ & $2.11 \pm 0.55^{*}$ & $2.18 \pm 0.76$ \\
\hline Transforming growth factor alpha & $2.78 \pm 0.49$ & $2.70 \pm 0.58$ & $2.60 \pm 0.50$ \\
\hline Furin & $3.07 \pm 0.36$ & $2.96 \pm 0.38$ & $3.12 \pm 0.41$ \\
\hline Protein CYR61 & $4.27 \pm 0.53$ & $4.23 \pm 0.51$ & $4.33 \pm 0.65$ \\
\hline Kallikrein-14 & $5.46 \pm 0.65$ & $5.31 \pm 0.67$ & $5.19 \pm 0.70$ \\
\hline FAS-associated death domain protein & $2.06 \pm 0.77$ & $1.66 \pm 0.73$ & $1.22 \pm 0.52^{*}$ \\
\hline Methionine aminopeptidase 2 & $2.85 \pm 0.87$ & $2.79 \pm 0.83^{*}$ & $2.44 \pm 0.61^{*}$ \\
\hline Nectin-4 & $5.52 \pm 0.35$ & $5.40 \pm 0.32^{*}$ & $5.25 \pm 0.49$ \\
\hline Tumor necrosis factor ligand superfamily member 6 & $8.72 \pm 0.36$ & $8.67 \pm 0.37$ & $8.74 \pm 0.44$ \\
\hline Ephrin type-A receptor 2 & $1.11 \pm 0.30$ & $1.08 \pm 0.32$ & $1.06 \pm 0.31$ \\
\hline Integrin beta-5 & $6.33 \pm 0.25$ & $6.27 \pm 0.26$ & $6.28 \pm 0.28$ \\
\hline Galectin-1 & $4.76 \pm 0.21$ & $4.76 \pm 0.21$ & $4.68 \pm 0.31$ \\
\hline Seizure 6-like protein & $4.46 \pm 0.24$ & $4.46 \pm 0.28$ & $4.45 \pm 0.29$ \\
\hline Transmembrane glycoprotein NMB & $5.38 \pm 0.16$ & $5.36 \pm 0.19$ & $5.37 \pm 0.18$ \\
\hline Carbonic anhydrase 9 & $1.66 \pm 0.56$ & $1.77 \pm 0.55$ & $1.69 \pm 0.75$ \\
\hline Melanoma-derived growth regulatory protein & $9.32 \pm 0.26$ & $9.31 \pm 0.26$ & $9.26 \pm 0.28$ \\
\hline Cathepsin L2 & $2.80 \pm 0.37$ & $2.59 \pm 0.30^{*}$ & $2.59 \pm 0.44$ \\
\hline
\end{tabular}


Table 2 (continued)

\begin{tabular}{|c|c|c|c|}
\hline Variable & Fasting $(n=22)$ & $\begin{array}{l}30 \text { min after food } \\
\text { intake }(n=21)\end{array}$ & $\begin{array}{l}120 \text { min } \\
\text { after food intake } \\
(n=22)\end{array}$ \\
\hline CD27 antigen & $7.52 \pm 0.34$ & $7.46 \pm 0.34$ & $7.50 \pm 0.49$ \\
\hline Xaa-Pro aminopeptidase 2 & $5.96 \pm 0.47$ & $5.90 \pm 0.47$ & $5.98 \pm 0.53$ \\
\hline Receptor tyrosine-protein kinase erbB-4 & $4.37 \pm 0.23$ & $4.31 \pm 0.25$ & $4.36 \pm 0.34$ \\
\hline Hepatocyte growth factor & $6.15 \pm 0.54$ & $6.06 \pm 0.50$ & $6.05 \pm 0.52$ \\
\hline Disintegrin and metalloproteinase domain-containing protein 8 & $4.10 \pm 0.33$ & $4.00 \pm 0.29$ & $3.96 \pm 0.39$ \\
\hline 5'-Nucleotidase & $9.05 \pm 0.31$ & $8.95 \pm 0.28$ & $9.00 \pm 0.34$ \\
\hline Cyclin-dependent kinase inhibitor 1 & $0.52 \pm 0.49$ & $0.42 \pm 0.48$ & $0.21 \pm 0.56$ \\
\hline Delta-like protein 1 & $8.60 \pm 0.31$ & $8.57 \pm 0.27$ & $8.59 \pm 0.34$ \\
\hline Midkine & $5.11 \pm 0.48$ & $4.82 \pm 0.63$ & $4.80 \pm 0.72$ \\
\hline Tyrosine-protein kinase ABL1 & $1.56 \pm 0.68$ & $1.31 \pm 0.69$ & $1.13 \pm 0.69$ \\
\hline Fibroblast growth factor-binding protein 1 & $6.21 \pm 0.26$ & $6.19 \pm 0.34$ & $6.10 \pm 0.37$ \\
\hline Toll-like receptor 3 & $4.68 \pm 0.57$ & $4.64 \pm 0.62$ & $4.68 \pm 0.68$ \\
\hline Tyrosine-protein kinase Lyn & $-0.06 \pm 0.19$ & $-0.15 \pm 0.21$ & $-0.19 \pm 0.25$ \\
\hline Proto-oncogene tyrosine-protein kinase receptor Ret & $4.30 \pm 0.47$ & $4.24 \pm 0.45$ & $4.29 \pm 0.43$ \\
\hline Vimentin & $3.68 \pm 0.37$ & $3.49 \pm 0.47$ & $3.32 \pm 0.49$ \\
\hline Tumor necrosis factor receptor superfamily member 19 & $3.95 \pm 0.41$ & $3.90 \pm 0.36$ & $3.81 \pm 0.54$ \\
\hline Cornulin & $5.44 \pm 1.16$ & $5.46 \pm 1.18$ & $5.51 \pm 0.99$ \\
\hline T-cell leukemia/lymphoma protein $1 \mathrm{~A}$ & $3.89 \pm 0.82$ & $3.78 \pm 0.82$ & $3.65 \pm 0.90$ \\
\hline CD160 antigen & $4.61 \pm 0.35$ & $4.55 \pm 0.28$ & $4.51 \pm 0.53$ \\
\hline Tumor necrosis factor receptor superfamily member 4 & $2.57 \pm 0.40$ & $2.51 \pm 0.36$ & $2.51 \pm 0.45$ \\
\hline MHC class I polypeptide-related sequence A and B & $2.60 \pm 1.78$ & $2.49 \pm 1.80$ & $2.56 \pm 1.85$ \\
\hline WNT1-inducible-signaling pathway protein 1 & $4.58 \pm 0.60$ & $4.48 \pm 0.64$ & $4.51 \pm 0.59$ \\
\hline VEGF-co regulated chemokine 1 & $1.69 \pm 0.46$ & $1.69 \pm 0.51$ & $1.70 \pm 0.61$ \\
\hline Pancreatic prohormone & $2.39 \pm 0.79$ & $4.29 \pm 0.91^{*}$ & $3.71 \pm 1.22^{*}$ \\
\hline Protein S100-A11 & $2.73 \pm 0.36$ & $2.62 \pm 0.36$ & $2.56 \pm 0.46$ \\
\hline Amphiregulin & $1.28 \pm 0.46$ & $1.26 \pm 0.37$ & $1.21 \pm 0.41$ \\
\hline Endothelial cell-specific molecule 1 & $7.99 \pm 0.48$ & $8.02 \pm 0.47$ & $8.09 \pm 0.52$ \\
\hline C-type lectin domain family 4 member $\mathrm{K}$ & $2.29 \pm 0.34$ & $2.22 \pm 0.30$ & $2.24 \pm 0.45$ \\
\hline ICOS ligand & $6.13 \pm 0.38$ & $6.00 \pm 0.46$ & $6.11 \pm 0.39$ \\
\hline WAP four-disulfide core domain protein 2 & $6.38 \pm 0.32$ & $6.36 \pm 0.30$ & $6.32 \pm 0.40$ \\
\hline C-X-C motif chemokine 13 & $8.26 \pm 0.57$ & $8.20 \pm 0.51$ & $8.32 \pm 0.60$ \\
\hline Mothers against decapentaplegic homolog 5 & $1.76 \pm 0.46$ & $1.80 \pm 0.55$ & $1.78 \pm 0.56$ \\
\hline A disintegrin and metalloproteinase with thrombospondin motifs 15 & $0.35 \pm 0.43$ & $0.45 \pm 0.42$ & $0.35 \pm 0.52$ \\
\hline CD70 antigen & $2.84 \pm 0.35$ & $2.87 \pm 0.38$ & $2.90 \pm 0.55$ \\
\hline R-spondin-3 & $-0.20 \pm 0.36$ & $-0.28 \pm 0.36$ & $-0.07 \pm 0.53$ \\
\hline Folate receptor gamma & $6.51 \pm 1.69$ & $6.48 \pm 1.75$ & $6.44 \pm 1.75$ \\
\hline Carcinoembryonic antigen-related cell adhesion molecule 5 & $1.45 \pm 0.62$ & $1.38 \pm 0.70$ & $1.48 \pm 0.64$ \\
\hline Vascular endothelial growth factor receptor 3 & $5.56 \pm 0.25$ & $5.54 \pm 0.25$ & $5.51 \pm 0.31$ \\
\hline Mucin-16 & $3.07 \pm 0.85$ & $3.12 \pm 0.78$ & $3.14 \pm 0.92$ \\
\hline Wnt inhibitory factor 1 & $5.23 \pm 0.47$ & $5.12 \pm 0.42$ & $5.16 \pm 0.50$ \\
\hline Granzyme B & $1.91 \pm 0.81$ & $1.75 \pm 0.86$ & $1.85 \pm 0.90$ \\
\hline Fc receptor-like B & $-0.39 \pm 0.55$ & $-0.33 \pm 0.55$ & $-0.38 \pm 0.57$ \\
\hline Annexin A1 & $1.93 \pm 0.43$ & $1.82 \pm 0.47$ & $1.69 \pm 0.50$ \\
\hline Folate receptor alpha & $5.83 \pm 0.29$ & $5.79 \pm 0.28$ & $5.81 \pm 0.43$ \\
\hline
\end{tabular}

All values are in arbitrary units (Mean $\pm S D$ )

* Indicates significant difference $(P<0.001)$, compared to fasting values 


\section{Abbreviations}

BMI: body mass index; AU: arbitrary units; SD: standard deviation; BSA: body surface area.

\section{Authors' contributions}

The authors' contributions were as follows: All authors designed the study. $\mathrm{JH}$ was responsible for recruiting the subjects. MD carried out the statistica calculations and wrote the first draft of the manuscript. JH and OB made critical revisions of the manuscript. All authors read and approved the final manuscript.

\section{Author details}

${ }^{1}$ Department of Medical Imaging and Physiology, Skåne University Hospital, Lund University, 20502 Malmö, Sweden. ${ }^{2}$ Department of Clinical Sciences, Division of Medicine, Skåne University Hospital, Lund University, Malmö, Sweden

\section{Acknowledgements}

Not applicable.

\section{Competing interests}

The authors declare that they have no competing interests.

\section{Availability of data and materials}

The dataset used during the current study are available from the corresponding author on reasonable request.

\section{Consent for publication}

Not applicable.

\section{Ethics approval and consent to participate}

All subjects gave their written informed consent to participate and consent for publication.

The study was approved the Regional Ethical Review Board in Lund, Sweden.

\section{Funding}

Nothing to declare.

\section{Publisher's Note}

Springer Nature remains neutral with regard to jurisdictional claims in published maps and institutional affiliations.

Received: 27 November 2018 Accepted: 28 March 2019

Published online: 02 April 2019

\section{References}

1. Global Burden of Disease Study 2013 Collaborators. Global, regional, and national incidence, prevalence, and years lived with disability for 301 acute and chronic diseases and injuries in 188 countries, 1990-2013: a systematic analysis for the Global Burden of Disease Study 2013. Lancet. 2013:386(9995):743-800.

2. Hlebowicz J, Lindstedt S, Björgell O, Dencker M. Relationship between postprandial changes in cardiac function glucose and insulin concentrations, gastric emptying, and satiety. Nutr J. 2011;10:26.

3. Hlebowicz J, Lindstedt S, Björgell O, Dencker M. The effect of endogenously released glucose, insulin, glucagon-like peptide 1, ghrelin on cardiac output, heart rate, stroke volume, and blood pressure. Cardiovasc Ultrasound. 2011;9:43.

4. Dencker M, Björgell O, Hlebowicz J. Effect of food intake on commonly used pulsed-Doppler and tissue-Doppler measurements. Echocardiography. 2011;28:843-7.

5. Dieden A, Gårdinger Y, Hlebowicz J, Bjorgell O, Dencker M. Effect of food intake on systolic tissue Doppler measurements. Clin Physiol Funct Imaging. 2016;36:396-400.

6. Gårdinger Y, Bjorgell $\mathrm{O}$, Hlebowicz J, Dencker M. Effect of food intake on left ventricular wall stress. Cardiovasc Ultrasound. 2014;12:2.
7. Quatela A, Callister R, Patterson A, MacDonald-Wicks L. The Energy content and composition of meals consumed after an overnight fast and their effects on diet induced thermogenesis: a systematic review, metaanalyses and meta-regressions. Nutrients. 2016;8(11):E670.

8. Dencker M, Gårdinger Y, Björgell O, Hlebowicz J. Effect of food intake on 92 biomarkers for cardiovascular disease. PLoS ONE. 2017;12(6):e0178656.

9. Dencker M, Gårdinger Y, Bjorgell O, Hlebowicz J. Effect of food intake on biomarkers for cardiovascular disease with the Proseek Multiplex CVD II kit. Genet Mol Res. 2018;17(1):16039884.

10. Dencker M, Bjorgell O, Hlebowicz J. Effect of food intake on 92 neurological biomarkers. Brain Behav. 2017;7(9):e00747.

11. Lundberg M, Eriksson A, Tran B, Assarsson E, Fredriksson S. Homogeneous antibody-based proximity extension assays provide sensitive and specific detection of low-abundant proteins in human blood. Nucleic Acids Res. 2011;39:e102.

12. Assarsson $E$, Lundberg $M$, Holmquist $G$, Björkesten J, Thorsen SB, Ekman D, Eriksson A, Rennel Dickens E, Ohlsson S, Edfeldt G, Andersson AC, Lindstedt P, Stenvang J, Gullberg M, Fredriksson S. Homogenous 96-plex PEA immunoassay exhibiting high sensitivity, specificity, and excellent scalability. PLoS ONE. 2014;9:e95192.

13. Enroth S, Johansson A, Enroth SB, Gyllensten U. Strong effects of genetic and lifestyle factors on biomarker variation and use of personalized cutoffs. Nat Commun. 2014;5:4684.

14. Enroth S, Bosdotter Enroth S, Johansson $\AA$, Gyllensten U. Effect of genetic and environmental factors on protein biomarkers for common noncommunicable disease and use of personally normalized plasma protein profiles (PNPPP). Biomarkers. 2015;20(6-7):355-64.

15. Chen H, Zucknick M, Werner S, Knebel P, Brenner H. Head-to-head comparison and evaluation of 92 plasma protein biomarkers for early detection of colorectal cancer in a true screening setting. Clin Cancer Res. 2015:21(14):3318-26.

16. Mahboob S, Ahn SB, Cheruku HR, Cantor D, Rennel E, Fredriksson S, Edfeldt G, Breen EJ, Khan A, Mohamedali A, Muktadir MG, Ranganathan S, Tan SH, Nice E, Baker MS. A novel multiplexed immunoassay identifies CEA, IL-8 and prolactin as prospective markers for Dukes'stages A-D colorectal cancers. Clin Proteomics. 2015;12(1):10.

17. Edfeldt K, Daskalakis K, Bäcklin C, Norlén O, Tiensuu Janson E, Westin G, Hellman P, Stålberg P. DcR3, TFF3 and Midkine are novel serum biomarkers in small intestinal neuroendocrine tumors. Neuroendocrinology. 2017;105(2):170-81

18. Loskog A, Maleka A, Mangsbo S, Svensson E, Lundberg C, Nilsson A, Krause J, Agnarsdóttir M, Sundin A, Ahlström H, Tötterman TH, Ullenhag G. Immunostimulatory AdCD40L gene therapy combined with lowdose cyclophosphamide in metastatic melanoma patients. Br J Cancer. 2016;114(8):872-80

19. Mailankody S, Devlin SM, Korde N, Lendvai N, Lesokhin A, Landau H, Hassoun H, Ballagi A, Ekman D, Chung DJ, Patel M, Koehne G, Giralt S, Landgren O. Proteomic profiling in plasma cell disorders: a feasibility study. Leuk Lymphoma. 2017;58(7):1757-9.

20. Schneiderova P, Pika T, Gajdos P, Fillerova R, Kromer P, Kudelka M, Minarik J, Papajik T, Scudla V, Kriegova E. Serum protein fingerprinting by PEA immunoassay coupled with a pattern-recognition algorithms distinguishes MGUS and multiple myeloma. Oncotarget. 2016:8(41):69408-21.

21. Söderlund S, Christiansson L, Persson I, Hjorth-Hansen H, Richter J, Simonsson B, Mustjoki S, Olsson-Strömberg U, Loskog A. Plasma proteomics in CML patients before and after initiation of tyrosine kinase inhibitor therapy reveals induced Th1 immunity and loss of angiogenic stimuli. Leuk Res. 2016;50:95-103.

22. Khandekar N, Berning BA, Sainsbury A, Lin S. The role of pancreatic polypeptide in the regulation of energy homeostasis. Mol Cell Endocrinol. 2015;418(Pt 1):33-41.

23. Joeckel LT, Bird PI. Blessing or curse? Proteomics in granzyme research. Proteomics Clin Appl. 2014;8(5-6):351-81.

24. Folkman J. Angiogenesis in cancer, vascular, rheumatoid and other disease. Nat Med. 1995;1(1):27-31.

25. Falguières T, Castle $D$, Gruenberg J. Regulation of the MVB pathway by SCAMP3. Traffic. 2012;13(1):131-42.

26. Suárez-Arroyo IJ, Feliz-Mosquea YR, Pérez-Laspiur J, Arju R, Giashuddin S, Maldonado-Martínez G, Cubano LA, Schneider RJ, Martínez-Montemayor $\mathrm{MM}$. The proteome signature of the inflammatory breast cancer plasma 
membrane identifies novel molecular markers of disease. Am J Cancer Res. 2016;6(8):1720-40.

27. Cimino Y, Costes A, Damotte D, Validire P, Mistou S, Cagnard N, Alifano $M$, Régnard JF, Chiocchia G, Sautès-Fridman C, Tourneur L. FADD protein release mirrors the development and aggressiveness of human nonsmall cell lung cancer. Br J Cancer. 2012;106(12):1989-96.

28. Pattje WJ, Melchers LJ, Slagter-Menkema L, Mastik MF, Schrijvers ML, Gibcus JH, Kluin PM, Hoegen-Chouvalova O, van der Laan BF, Roodenburg JL, van der Wal JE, Schuuring E, Langendijk JA. FADD expression is associated with regional and distant metastasis in squamous cell carcinoma of the head and neck. Histopathology. 2013;63(2):263-70.

29. Jahn LA, Hartline L, Rao N, Logan B, Kim JJ, Aylor K, Gan LM, Westergren $\mathrm{HU}$, Barrett EJ. Insulin enhances endothelial function throughout the arterial tree in healthy but not metabolic syndrome subjects. J Clin Endocrinol Metab. 2016;101(3):1198-206.

30. McFarlin BK, Carpenter KC, Henning AL, Venable AS. Consumption of a high-fat breakfast on consecutive days alters preclinical biomarkers for atherosclerosis. Eur J Clin Nutr. 2017;71(2):239-44.
Ready to submit your research? Choose BMC and benefit from:

- fast, convenient online submission

- thorough peer review by experienced researchers in your field

- rapid publication on acceptance

- support for research data, including large and complex data types

- gold Open Access which fosters wider collaboration and increased citations

- maximum visibility for your research: over $100 \mathrm{M}$ website views per year

At BMC, research is always in progress.

Learn more biomedcentral.com/submissions 